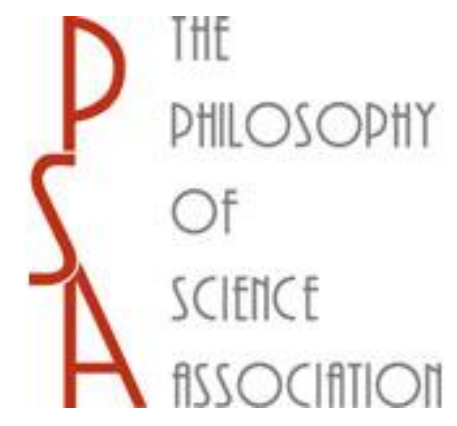

Environmental Risk Analysis: Robustness Is Essential for Precaution

Author(s): Jan Sprenger

Source: Philosophy of Science, Vol. 79, No. 5 (December 2012), pp. 881-892

Published by: The University of Chicago Press on behalf of the Philosophy of Science Association

Stable URL: https://www.jstor.org/stable/10.1086/667873

JSTOR is a not-for-profit service that helps scholars, researchers, and students discover, use, and build upon a wide range of content in a trusted digital archive. We use information technology and tools to increase productivity and facilitate new forms of scholarship. For more information about JSTOR, please contact support@jstor.org.

Your use of the JSTOR archive indicates your acceptance of the Terms \& Conditions of Use, available at https://about.jstor.org/terms

The University of Chicago Press and Philosophy of Science Association are collaborating with JSTOR to digitize, preserve and extend access to Philosophy of Science 


\title{
Environmental Risk Analysis: Robustness Is Essential for Precaution
}

\author{
Jan Sprenger*†
}

Precaution is a relevant and much-invoked value in environmental risk analysis, as witnessed by the ongoing vivid discussion about the precautionary principle (PP). This article argues (i) against purely decision-theoretic explications of PP; (ii) that the construction, evaluation, and use of scientific models falls under the scope of PP; and (iii) that epistemic and decision-theoretic robustness are essential for precautionary policy making. These claims are elaborated and defended by means of case studies from climate science and conservation biology.

1. Introduction: Science, Policy, and Precaution. The interplay of environmental science and public policy is nowadays the subject of vivid discussion, especially in environmental risk analysis. Conservation biologists make up lists of endangered and acutely threatened species. Environmental economists study the effects of specific environmental policies on issues such as water quality, air pollution, or toxic waste. Most prominently, climate scientists advise policy makers about the causes and consequences of global warming (e.g., IPCC 2007).

In these contexts, a precautionary attitude is often of great relevance. For instance, the participants of the UN summit in Rio de Janeiro (1992) stated in their final declaration: "In order to protect the environment, the precautionary approach shall be widely adopted by States according to their capabilities. Where there are threats of serious or irreversible damage, lack of full

\footnotetext{
* To contact the author, please write to: Tilburg Center for Logic and Philosophy of Science, Tilburg University, PO Box 90153, 5000 LE Tilburg, The Netherlands; e-mail: j.sprenger@ uvt.nl.

$†$ For helpful discussion and feedback, I would like to thank Mark Burgman, Mark Colyvan, Sven Ove Hansson, Stephan Hartmann, James Justus, Martin Peterson, Helen Regan, Moshe Sniedovich, Katie Steele, Arie Trouwborst, Jonathan Verschuuren, and the numerous audiences where this work was presented. Research on this topic was financially supported by Veni grant 016.104.079 by the Netherlands Organisation for Scientific Research.
}

Philosophy of Science, 79 (December 2012) pp. 881-892. 0031-8248/2012/7905-0026\$10.00

Copyright 2012 by the Philosophy of Science Association. All rights reserved. 
scientific certainty shall not be used as a reason for postponing cost-effective measures to prevent environmental degradation" (United Nations 1993).

But what does the demand for precautionary policy imply for environmental science? As a matter of fact, a precautionary approach is often required or recommended for studies on ecological risks of economic developments, assessments of the survival chances of endangered species, or biosecurity risk assessments. For example, in the annex to the criteria for classifying endangered species, the International Union for Conservation of Nature states that "assessors should . . . adopt a precautionary but realistic attitude to uncertainty when applying the [classification] criteria, for example, by using plausible lower bounds, rather than best estimates, in determining population size" (IUCN 2000, 25). In this context, using lower bounds instead of best estimates is a natural way of implementing precaution into one's analysis. However, assessment problems are often more complex than such simple estimation problems, and it is unclear what a "precautionary attitude" in scientific modeling really amounts to.

This article analyzes the role of precaution in environmental risk analysis. More specifically, it tries to explicate the precautionary principle (PP), which plays a prominent role in environmental law and environmental policy making. Thus, the topic is relevant from both a philosophical and a policy perspective.

The article is structured as follows: first, I argue, building on the existing literature, that explications of PP as a specific decision rule or as an alternative to standard decision theory are bound to fail (sec. 2). Second, I transfer PP to questions of model construction, evaluation, and use in the environmental sciences. Third, I elaborate the main thesis - robustness is an essential part of a precautionary attitude - from two case studies, one in climate science (sec. 3) and one in conservation biology (sec. 4). I conclude by summarizing my findings, sketching their implications, and responding to objections (sec. 5).

2. Explicating the Precautionary Principle. The PP - a successor of the German Vorsorgeprinzip - intends to guide decision making in the face of environmental hazards and scientific uncertainty. It has, by now, achieved a prominent position in environmental policy, as witnessed by its inclusion in international treaties, declarations, and legal systems (Trouwborst 2006). Its applications may include cases as diverse as approving of the exploitation of natural resources, building a pipeline through a natural reserve, constructing atomic power plants, and so on.

But what exactly does the principle state? A few years after the aforementioned Rio summit, the Wingspread Conference gathered environmentalists, scientists, lawyers, and policy makers who agreed on the following formulation of PP: "When an activity raises threats of harm to human health or the 
environment, precautionary measures should be taken even if some cause and effect relationships are not fully established scientifically. In this context the proponent of an activity, rather than the public, should bear the burden of proof" (Wingspread Conference on the Precautionary Principle, 1998; quoted at http://www.sehn.org/wing.html). This wording is somewhat stronger than the one agreed on at the Rio summit: now, it is about actively taking precautionary measures, not about whether scientific uncertainty is a valid reason for inactivity. PP now imposes the requirement to refrain from actions and policies that run a risk of causing harm to the public or to the environment. Notably, it is not required that science has established the harmfulness of such actions beyond a reasonable doubt (e.g., Sandin 1999; Wiener et al. 2010).

Precaution supposedly applies at the final, decision-making stage of the analysis - after science has informed policy makers about the potential environmental hazards. It is about approving or not approving of a certain activity. In that understanding, the $\mathrm{PP}$ is akin to a first-order decision rule: a rule for transforming an assessment of uncertainties and potential losses into a final decision (e.g., Hansson 1997).

Several interpretations of PP — especially by its critics - presuppose such a reading and let it compete with decision rules such as expected utility maximization, maximin, and the like. These explications often focus on the probability of a fatal outcome:

$\mathbf{P P}_{\alpha}$. If one act is more likely to give rise to a fatal outcome than another, then the latter should be preferred to the former.

$\mathbf{P P}_{\beta}$. If one act is more likely to give rise to a fatal outcome than another and if both fatal outcomes are equally undesirable, then the latter should be preferred to the former.

Peterson (2006) shows convincingly that these decision rules conflict with attractive principles of rational choice. Let us assume (1) that preferences are totally ordered, that is, they are complete, asymmetric and transitive; (2) that our decision rules obey dominance reasoning; and (3) that shifting probability from bad to good outcomes can only increase the desirability of an action. ${ }^{1}$ Then, $\mathrm{PP}$ is in either explication $\left(\mathrm{PP}_{\alpha}\right.$ or $\left.\mathrm{PP}_{\beta}\right)$ logically incompatible with these three principles.

The source of the problem is the intuition that both the probability and the desirability/potential harm of an outcome matter and that they can, to some

1. The assumptions in principle 1 are perhaps not too realistic for real agents, but they should hold for idealized rational agents. Since we are interested in how PP supports rational decisions, we retain these assumptions. 
extent, be traded off against each other. This view is deeply entrenched in most accounts of rational decision making, but it conflicts with the above explications of PP. More precisely, $\mathrm{PP}_{\alpha}$ and $\mathrm{PP}_{\beta}$ oppose the view that if we (marginally) increase the likelihood of a fatal outcome, we can counterbalance this risk by (substantially) increasing the likelihood of a favorable outcome. Peterson also investigates refinements of $\mathrm{PP}_{\alpha}$ and $\mathrm{PP}_{\beta}$ but concludes that they suffer from the same problem.

Apart from these decision-theoretic concerns, such a reading of the PP is also unrealistically conservative. That is, it focuses exclusively on the possibility of disasters and makes effective action virtually impossible (Harris and Holm 1999). For some discoveries, like new medical drugs, it is impossible to rule out adverse effects a priori; nevertheless, the benefits of a successful innovation can sometimes be so great that it seems to be reasonable to take the risk. Indeed, the consequences of such a reading of the PP would be so hostile to innovation that one may charge the critics with basing their points on a very uncharitable interpretation. ${ }^{2}$

Moreover, the difficulty of specifying meaningful, empirically based subjective probabilities of extreme, harmful events (such as "runaway climate change in a scenario without $\mathrm{CO}_{2}$ abatement") speaks against an interpretation as a probabilistic decision rule. This concern is particularly outspoken in climate science, where the ability of climate models to support subjective probability assessments as a basis for decision making is often problematized (e.g., Frame et al. 2007).

Since explications of PP as a concrete decision rule are inadequate for the above reasons, the more recent literature (e.g., Steele 2006) remains skeptical about concrete decision-theoretic implications of PP. Efforts have shifted to aligning PP with a method for building decision models or specific goals of a risk analysis. For example, the COMEST (World Commission on the Ethics of Scientific Knowledge and Technology) report on the PP states that "[when] ethical dimensions of inter- and intragenerational equity are at stake, the other decision principles [such as expected utility maximization] fail to satisfactorily address these problems characteristics. . . . Because the PP applies to these cases where serious adverse effects/surprises can occur with unknown probability, it is rational to follow a 'better safe than sorry' strategy" (COMEST 2005, 30; my emphasis). In the spirit of the COMEST report, Steele $(2006,29)$ surmises that PP might be best understood as (i) urging us to thoroughly survey the decision space (herein she follows

2. Another argument against a narrow decision-theoretic reading claims that standard decision theory, such as expected utility theory, already has plenty of resources to account for risk aversion in trading off likelihoods and payoffs. This point was brought to my attention by Franz Dietrich, Christian List, and Kai Spiekermann at a recent meeting in Munich. 
Resnik [1987]) and (ii) promoting "an ethical outlook consistent with the ideal of sustainable development," giving intra- and intergenerational equity a "significant influence [on] the evaluation of act outcomes." Thus, Steele focuses on the ends to pursue rather than the means to achieve these ends.

Carefully surveying the decision space is certainly a useful aspect of precautionary policy making, but it does not explain why PP does and should play such a prominent role in environmental risk analysis. After all, a careful survey is a good thing to do in almost any decision problem. Moreover, it may be doubted that the PP is primarily about the ethical goals that one should have, rather than about how to reach these goals in the face of uncertainty. Therefore, the readings proposed by Resnik and Steele do not constitute a fully convincing explication of PP. This prompts the legitimate question of whether we could better drop PP altogether: if we cannot give it a concrete meaning, the principle may be more confusing than helpful.

In reply to this challenge, I contend that precaution in environmental risk analysis has substantial implications for the way scientific models are built, evaluated, and used. More precisely, I argue that robustness is, both in its epistemic and its decision-theoretic form, a key element of precautionary risk analysis. These different facets of robustness are elucidated by means of two case studies to which the next two sections are devoted.

3. Case Study 1: Climate Models and Climate Policy. In the economics of climate change, there is a classic and very influential analysis by Nordhaus (1991). He argues on the basis of a specific economic model that reducing greenhouse gas emissions may be an economically inefficient strategy: for low- and medium-damage functions of climate change, very little reduction will be the best strategy. Only for high-damage functions, a substantial reductions of greenhouse gas emissions (about 33\%) will be the best option.

Intuitively, this analysis sounds highly optimistic, given the range of scientifically possible scenarios in which substantial, irreversible damage occurs (e.g., through a runaway climate change). As Nordhaus admits himself, his analysis is based on a lot of contentious simplifications with respect to quantifying the costs of climatic change and on value-laden assumptions about discount rates. The modeling of natural disasters resulting from global warming and their complex economic consequences remains extremely rough: some natural hazards (e.g., more hurricanes and flooding in the United States) are incorporated into the model, while others, such as economic imbalances and political tensions due to water shortage in more and more regions of the world, are completely neglected. Indeed, more recent studies like Stern (2007) reach the opposite result, concluding that the benefits of immediate $\mathrm{CO}_{2}$ abatement clearly outweigh the costs.

But even if Nordhaus's model were widely recognized as the best model of the economics of climate change, we could still argue that the suggested 
policy conclusion - relatively little $\mathrm{CO}_{2}$ abatement - is not sufficiently precautionary. Given the different scale, target, and assumptions of models in climate science and climate economics, any model can only provide incomplete scientific knowledge and partially justify policy decisions. It would then violate the precautionary approach to derive environmental policy directly from the conclusions of a particular, simplistic model, rather than from a meta-analysis of various models.

In other words, to rationally defend nonabatement as a precautionary policy, one would require that most models assess the costs of mitigation as significantly higher than the costs of adaptation. After all, while we have some control over the economic costs of mitigation, we might not be able to control the costs of unmitigated global warming. But the emerging consensus in climate economics rather suggests the contrary: immediate mitigation is, in the long run, a way more efficient and less costly strategy than pure adaptation.

This small example suggests that we might best understand precaution in terms of robustness. An act that allows for certain environmental hazards has duly taken into account precautionary considerations if diverse, structurally different models indicate that these hazards may be negligible. Structural differences may occur along the dimensions of target system, scale, physical motivation, time horizon, and so on. This reading of PP can be defended especially well if no single best model is available such that the analysis has to rely on the results of diverse models. In climate science and climate economics, these conditions are more often than not satisfied.

To some extent, this view is supported by the focus on multimodel ensembles in climate science: "averages across structurally different models empirically show better agreement with observations because individual model biases tend to cancel" (IPCC 2007, working group 1, 754; see also Tebaldi and Knutti 2007). This response to the large uncertainty in climate modeling is also inspired by robustness considerations and constitutes a substantial shift from the traditional "best-model approach" in which the most reliable model was used for prediction and risk assessment. ${ }^{3}$

Figure 1, taken from the IPCC's 4th Assessment Report, presents various probability density functions for climate sensitivity - the degree to which the temperature of the climate system is responsive to a change in radiative forcing, and in particular to a doubling of $\mathrm{CO}_{2}$ concentration in the atmosphere, relative to a baseline level. We can see quite clearly that there are substantial

3. Accounting for uncertainty by means of hierarchical models or second-order probabilities is similar to straightforward model averaging, in the sense that conclusions are reached by averaging the models according to the prespecified second-order uncertainties. While this strategy certainly improves on basing conclusions on the best guess, it is also less precautionary than a full-fledged robustness analysis. 


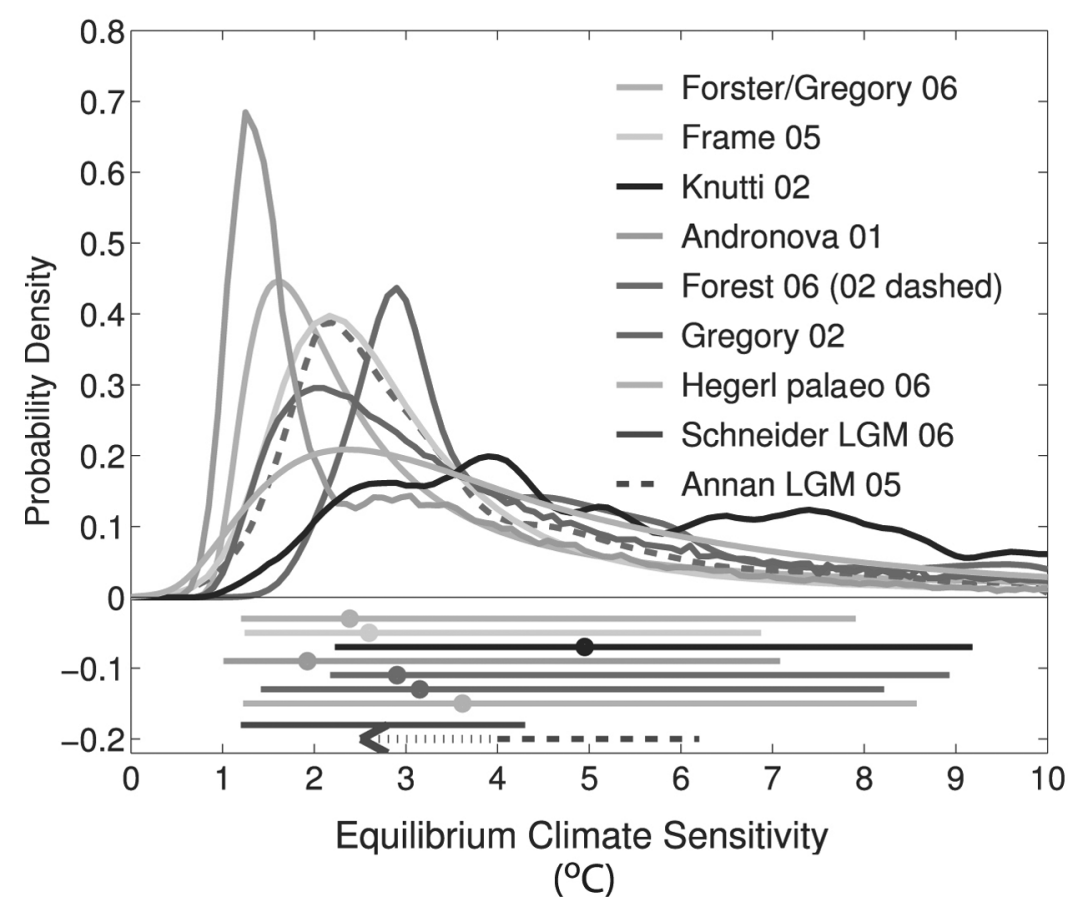

Figure 1. Probability density functions for equilibrium climate sensitivity in various climate models. Source: IPCC (2007), working group 1, fig. 9.20. Color version available as an online enhancement.

differences between the models, concerning both the mean of the distribution and the width of the confidence intervals. It would not make much sense to use a point estimate or a narrow confidence interval as a basis for further analysis and policy making. Instead, a precautionary attitude has to appreciate the uncertainty about the true value of climate sensitivity and the divergence between the individual models.

This emphasis on robustness, as well as the distinction between confidence (within a model) and consistency (across models), is also reflected in the practice of scientific policy advice. The synthesis of the 4th Assessment Report of the IPCC (2007) contains a separate chapter (chap. 6) in which the most important conclusions that are robust across various models are summarized and quantified by means of a confidence level. For example, "equilibrium climate sensitivity is very unlikely to be less than $1.5^{\circ} \mathrm{C}$." The fact that this is the unanimous result of structurally different models increases our confidence that the value of equilibrium climate sensitivity will indeed be greater than $1.5^{\circ} \mathrm{C}$, whereas for a single model, all kinds of uncertainties may 
invalidate the conclusion, and individual model biases cannot cancel out one another. $^{4}$

Thus, rather than a straightforward principle of decision making, precaution in environmental risk analysis becomes a matter of assessing and responding to model uncertainty. And since precaution is sensitive to how much agreement between structurally different studies is observed, robustness becomes one of its essential elements.

4. Case Study 2: The Sumatran Rhino. The explication of PP sketched in the previous section does not apply when there are no competing models of a single phenomenon. In this case, the understanding of precaution as robustness has to differ from cross-model consistency. Rather, we have to look for ways to make our conclusions more robust within a single model. To illustrate this point, I present two different risk analyses for the Sumatran rhino conservation problem.

The original analysis (Maguire, Seal, and Brussard 1987) evaluated the available options - translocation, new reserve, and captive breeding - by means of ranking them according to their expected utility. In that model, the utility function is defined as a decreasing linear function of the probability of extinction in various states of the world. The probabilities for the possible hazards (poaching, loss of habitat, etc.) were elicited by means of expert judgment. A simplified summary of such an analysis is given in table $1 .{ }^{5}$

The act of "captive breeding" has the highest expected utility and was, in fact, the one recommended by the scientists and implemented by the policy makers, with disastrous consequences. Post mortem, this is not surprising since captive breeding is especially vulnerable to demographic accidents in the captive population, which quite likely is a hazard. Underestimating the probability of that event can have far-reaching effects for the expected utility of captive breeding. Unfortunately, environmental management problems are often characterized by substantial uncertainties, casting doubt on the accuracy of the probabilities and utilities that figure in the model.

To overcome these problems, Regan et al. (2005) conducted a reanalysis of the conservation problem and replaced the expected utility analysis by means of Yakov Ben-Haim's (2006) info-gap approach. The principal idea of the info-gap is borrowed from the radius-of-stability model in applied mathematics: it chooses the option that allows for the greatest deviation from an initial best estimate, while still satisfying a given performance threshold.

4. See Justus (2012) for a discussion of the confirmatory value of robustness in environmental science.

5. Table 1 is not a representation of the actual results of Maguire et al. (1987), but Regan et al. (2005) use this table to compare an expected utility perspective to their own model. I am following their analysis here. 
table 1. Utility Matrix of the Sumatran Rhino Conservation Problem

\begin{tabular}{lcccc}
\hline Option/State & Poaching & Loss of Habitat & Demographic Accidents & Disease \\
\hline Probability of state & .1 & .3 & .5 & .01 \\
Translocation & .3 & .1 & .05 & .1 \\
New reserve & .3 & .1 & .05 & .1 \\
Captive breeding & .9 & .2 & .01 & .4 \\
\hline
\end{tabular}

Note.-Data follow the analysis of Maguire et al. (1987).

To make this idea more precise, assume that we want to estimate an unknown function of the variable $t$ by an estimate $\hat{u}(t)$. The core idea is to define a info-gap model $\mathcal{U}(\hat{u}, \alpha)$ of the initial estimate, namely, the set of functions $u(t)$ such that the fractional deviation of $\hat{u}$ from $u$ (or another reasonable divergence measure, such as the $L^{2}$ norm) does not exceed a threshold $\alpha$ :

$$
\mathcal{U}(\hat{u}, \alpha)=\left\{u(t): \forall t:\left|\frac{u(t)-\hat{u}(t)}{\hat{u}(t)}\right| \leq \alpha\right\} .
$$

The threshold value $\alpha$ is here called the horizon of uncertainty: it reflects the quality of the initial estimate and determines which functions $u(t)$ are taken into account, dependent on their distance from the initial estimate. Any available option $q$ in the original decision problem can now be assessed in terms of the minimal return it ensures, for a given horizon of uncertainty $\alpha$. This assessment is conducted by giving the robustness function of a decision $q$ : the maximal horizon of uncertainty for which $q$ still yields an acceptable outcome, that is, a loss $L(q, u)$ below the critical value $l_{c}$ :

$$
\hat{\alpha}\left(q, l_{c}\right)=\max \left\{\alpha:\left(\max _{\mathrm{u} \in \mathcal{U}(\hat{\mathrm{u}}, \alpha)} L(q, u)\right) \leq l_{c}\right\} .
$$

In other words, $\hat{\alpha}$ quantifies the local robustness of a decision $q$ regarding uncertainty in the estimate $\hat{u}(t)$, by indicating the highest horizon of uncertainty for which a satisfactory decision (as measured by the loss function $L$ ) is always ensured.

In the case of the Sumatran rhino, the posthumous analysis of Regan et al. (2005) shows that an info-gap perspective would lead to a different decision. When the estimated probabilities and utilities in the original analysis are subjected to an info-gap analysis, we realize that captive breeding is superior to the other options for a narrow horizon of uncertainty only (see fig. 2). As soon as the uncertainty attached to the probabilistic estimates increases, captive breeding becomes more vulnerable to error, and we may be better advised to establish a new reserve. This decision may then justifiably be called a more robust choice. 


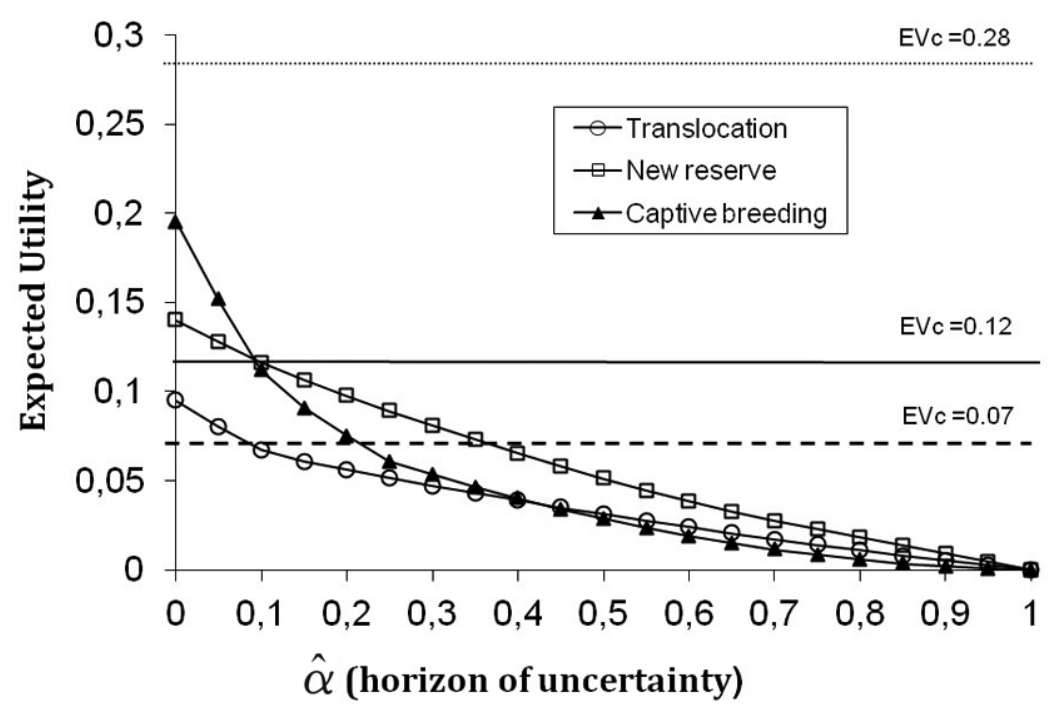

Figure 2. Minimal expected utility of the three management options in table 1, as a function of the horizon of uncertainty. Source: Regan et al. (2005).

This approach has a variety of virtues. By means of the horizon of uncertainty, robustness considerations are directly built into the model. Moreover, we can directly plug in our best scientific model and evaluate the estimates based on that model. However, the performance of the info-gap depends on the quality of the initial estimate. Therefore, the info-gap addresses the issue of local rather than global robustness; that is, it conducts a sensitivity analysis around the initial estimate. In particular, the info-gap does not take into account that the initial estimate may be grossly mistaken - then it would be clearly inadequate to maximize the horizon of uncertainty around that value (Sniedovich 2010).

All in all, the Sumatran rhino case study demonstrates how a precautionary attitude motivates a different approach to environmental risk analysis that improves on naive expected utility maximization. In this specific case, a more precautionary analysis (info-gap) focuses on the robustness of a best estimate under various forms of uncertainty. Again, a decision is just as precautionary as the estimates on which it is based are robust.

5. Discussion. The PP is a well-known principle for making environmental policy decisions on the basis of scientific findings. This article has argued that the construction, evaluation, and use of scientific models falls under the scope of PP, and it has investigated how a precautionary attitude in environmental risk analysis can be explicated in practice. 
After rejecting interpretations of PP as alternatives to standard decision theory, I have argued that a precautionary attitude in environmental risk analysis needs to engage with the involved scientific models. More specifically, I have proposed that epistemic and decision-theoretic robustness with respect to model building, evaluation, and use are essential to precautionary policy making.

A main argument for this understanding of precaution consisted in the observation that modeling in the environmental sciences often comes with substantial uncertainties. Therefore, robustness is especially important from a precautionary point of view. It comes in two varieties. One of them, epistemic robustness, enhances precaution by means of consistency across different models, like in meta-analysis. This is especially relevant when there are competing models of the problem of interest that are more or less on a par. Robustness considerations take into account the limited confidence that we have in any single model, expose the limitations of existing models, and suggest roads for future improvements.

By contrast, decision-theoretic robustness means that the superiority of an act is maintained if assumptions of the model are modified. This is especially relevant when there is only one reasonable model of the problem of interest but parameter values are highly uncertain. Robust decisions can be qualified as precautionary because their justifications are less demanding than decisions based on a less robust analysis. Both cases have been illustrated by case studies from climate science and conservation biology.

Before concluding, I discuss two possible objections to the main theses of this article. First, it could be argued that robustness is something that we would like to pursue anyway, that it is not specific to environmental decision making. But the knowledge basis for our decisions can change quickly, as the case of climate science convincingly illustrates. Thus, responsible decision making needs to conserve some skepticism about our current state of knowledge, even if it is informed by our best science. This speaks for making decisions on the basis of assumptions that hold in various scenarios. Moreover, especially in international contexts, policy decisions are difficult to overturn, given the lengthy and cost-intensive deliberations required to reach an agreement in the first place. For those reasons, robustness should play a central role in environmental risk analysis.

Second, given that PP has been developed as a principle for policy making, my proposal seems to give too much weight to the scientific dimension of precaution. Two counterarguments can be given: first, we have seen that explications of PP in terms of a principle that only applies to final decisions do not hold water. Second, recent research in philosophy of science (e.g., Douglas 2009) has argued that a strict division of (scientific) risk assessment and risk management (policy making) is unrealistic. 
Following up on that last point, we note that the details of applying PP in a concrete case inevitably demand scientific expertise beyond "providing the numbers." Precautionary environmental decisions demand an intense dialogue and joint efforts of scientists and policy makers, rather than separating the two domains.

\section{REFERENCES}

Ben-Haim, Yakov. 2006. Info-Gap Decision Theory: Decisions under Severe Uncertainty. 2nd ed. San Diego, CA: Academic Press.

COMEST (World Commission on the Ethics of Scientific Knowledge and Technology). 2005. The Precautionary Principle. Paris: UNESCO.

Douglas, Heather. 2009. Science, Policy, and the Value-Free Ideal. Pittsburgh: University of Pittsburgh Press.

Frame, D. J., N. E. Faull, M. M. Moshi, and M. R. Allen. 2007. "Probabilistic Climate Forecasts and Inductive Problems." Philosophical Transactions of the Royal Society A 365:1971-92.

Hansson, Sven Ove. 1997. "The Limits of Precaution." Foundations of Science 2:293-306.

Holm, Søren, and John Harris. 1999. "Precautionary Principle Stifles Discovery." Nature 400:398.

IPCC (Intergovernmental Panel on Climate Change). 2007. "4th Assessment Report.” IPCC. http:// www.ipcc.ch.

IUCN (International Union for Conservation of Nature). 2000. "The IUCN Redlist Categories and Criteria." Vers. 3.1. IUCN, Gland. http://www.iucn.org.

Justus, James. 2012. "The Elusive Basis of Inferential Robustness." Philosophy of Science, in this issue.

Maguire, L. A., U. S. Seal, and P. F. Brussard. 1987. "Managing Critically Endangered Species: A Case Study of the Sumatran Rhino." In Viable Populations for Conservation, ed. M. Soulé, 141-58. Cambridge: Cambridge University Press.

Nordhaus, William. 1991. "To Slow or Not to Slow: The Economics of the Greenhouse Effect." Economic Journal 101:920-37.

Peterson, Martin. 2006. “The Precautionary Principle Is Incoherent.” Risk Analysis 26:595-601.

Regan, H. M., Y. Ben-Haim, B. Langford, W. G. Wilson, P. Lundberg, S. J. Andelman, and M. A. Burgman. 2005. "Robust Decision Making under Severe Uncertainty for Conservation Management." Ecological Applications 15:1471-77.

Resnik, Michael D. 1987. Choices: An Introduction to Decision Theory. Minneapolis: University of Minnesota Press.

Sandin, Per. 1999. "Dimensions of the Precautionary Principle." Human and Ecological Risk Assessment 5:889-907.

Sniedovich, Moshe. 2010. “A Bird's View of Info-Gap Decision Theory.” Journal of Risk Finance $11: 268-83$

Steele, Katie. 2006. "The Precautionary Principle: A New Approach to Public Decision-Making?" Law, Probability and Risk 5:19-31.

Stern, Nicholas. 2007. The Economics of Climate Change: The Stern Review. Cambridge: Cambridge University Press.

Tebaldi, Claudia, and Reto Knutti. 2007. "The Use of the Multi-Model Ensemble in Probabilistic Climate Projections." Philosophical Transactions of the Royal Society A 365:2053-75.

Trouwborst, Arie. 2006. "Precautionary Rights and Duties of States." PhD diss., Utrecht University.

United Nations. 1993. Principle 15 of the Rio Declaration on Environment and Development. In Report of the United Nations Conference on the Human Environment, Stockholm, 5-16 June 1972. New York: United Nations. http://www.unep.org/Documents.multilingual/Default.asp ?DocumentID=78\&ArticleID=1163.

Wiener, Jonathan, Michael D. Rogers, James K. Hammitt, and Peter H. Sand. 2010. The Reality of Precaution. London: Taylor \& Francis. 